\title{
Learned Features are Better for Ethnicity Classification
}

\author{
Inzamam Anwar, Naeem Ul Islam \\ Intelligent Systems Research Institute (ISRI), College of Information and Communication Engineering, \\ Sungkyunkwan University, Suwon, South Korea \\ E-mails:inzimammanzoor@gmail.comnaeem@skku.edu
}

\begin{abstract}
Ethnicity is a key demographic attribute of human beings and it plays a vital role in automatic facial recognition and have extensive real world applications such as Human Computer Interaction (HCI); demographic based classification; biometric based recognition; security and defense to name a few. In this paper, we present a novel approach for extracting ethnicity from the facial images. The proposed method makes use of a pre trained Convolutional Neural Network (CNN) to extract the features, then Support Vector Machine (SVM) with linear kernel is used as a classifier. This technique uses translational invariant hierarchical features learned by the network, in contrast to previous works, which use hand crafted features such as Local Binary Pattern (LBP); Gabor, etc. Thorough experiments are presented on ten different facial databases, which strongly suggest that our approach is robust to different expressions and illuminations conditions. Here we consider ethnicity classification as a three class problem including Asian, African-American and Caucasian. Average classification accuracy over all databases is $98.28 \%$, 99.66\% and $99.05 \%$ for Asian, African-American and Caucasian respectively. All the codes are available for reproducing the results on request.
\end{abstract}

Keywords: Ethnicity recognition, race classification, Convolutional Neural Network $(C N N)$, VGG Face, Support Vector Machine (SVM).

\section{Introduction}

Ethnicity identification is the process of recognizing the ethnic group of an individual from a face image. Human face provides wealth of information including identity, gender, age, race, expression, etc. Among the demographic attributes of gender, age, ethnicity, etc., ethnicity remains invariant through all lifetime and greatly supports face recognition systems, therefore, automatic facial ethnicity classification has been received increasing attention in recent years and several methods have been proposed. However, accurate and swift classification of different races based on human face in an uncontrolled environment is challenging. For efficient classification, one has to find race sensitive features from face images. These discriminative features can be differentiated in three categories [1], namely: chromatic/skin tone, local features and 
global features. Due to similar skin colour for different races and extreme variation in illumination conditions for real world scenarios, skin tone alone cannot classify. However, combined with local or global descriptors classification accuracy can be boosted.

Feature based identification is based on feature extraction either globally or locally. In [2], a two class ethnicity classification for Asian and non-Asian images is presented. Rather than using full image, the method determine the confidence of different facial regions by employing modified Golden ration mask followed by using Support Vector Machine (SVM) classifier on facial features such as eyes, nose and mouth. Similarly, a two class ethnicity approach for classification based on Linear Discriminant Analysis (LDA) [3] and Principle Component Analysis [4] are also presented. Nevertheless, these methods are limited and it is unclear about the efficiency of these approaches in more realistic conditions of multi-ethnic groups. The LDA approach in [3] was later enhanced with geometric features extracted using Gabor wavelet transform [5] for a three-class scenario.

Relationship between iris texture and race group is studied by many researchers in past [1]. Results have shown that there is a strong relationship between iris texture and race [6-8]. However, due to low resolution and distant images of subjects in real world, one cannot rely on cumbersome method of iris detection and identification. $\mathrm{Lin}, \mathrm{Lu}$ and $\mathrm{Zh}$ an $\mathrm{g}$ [9] have used Adaboost and Gabor filters to extract features, which are classified using SVM and presented results on FERET database. Local Binary Pattern (LBP) is a non-parametric descriptor, which summarizes the local structures of images efficiently. In [10], demographic classification based on LBP is presented. In order to further improve the accuracy of race identification, various methods based on the fusion of different features are presented [11-13].

Recently deep learning methods have shown promising results in computer vision. With the breakthrough results in Large Scale Image Classification and Recognition Challenge (ILSVRC) [14], many researchers are using deep learning methods, especially Convolutional Neural Network (CNN), in different recognition and classification problems. These advancements in deep learning happen due to availability of more computational power and large datasets for training. Convolutional Neural Network consists of stack of layers, which learn the translational invariance and hierarchal contextual features from input images. This type of deep learning becomes popular and outperforms previous state of the art techniques in various domains [15-21].

This paper presents a framework to find ethnicity of the subject from face images using the deep CNN, pre-trained on large-scale face database. This network is used as an independent feature extractor, which are then classified with a linear classifier. Exhaustive experimentation is presented on ten databases. Results of our proposed approach strongly advocate that it is robust to different illumination conditions, expression, accessories, background, age and gender. The rest of the paper is organized in the following way: in Section 2, a brief introduction of CNN, VGG network and pre-processing is presented. Section 3 provides introduction of datasets used and presents results of in depth experimentation. In Section 4 conclusions from this study and experiments are drawn. 


\section{Proposed approach}

\subsection{Convolutional neural network}

CNN are derived from basic neural networks. In neural networks, there are three types of layer, input, hidden and output. Each layer have several neurons stacked in it, which takes input from the previous layers. In each layer, a neuron acts as a linear classifier. Each neuron performs some mathematical operation, usually computes a dot product of input with its respective weight, adds biasing, and applies a nonlinearity.

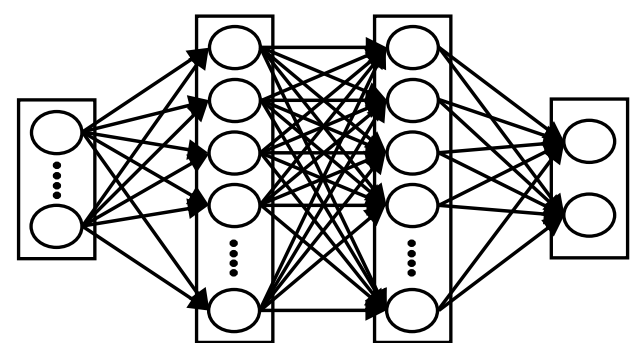

Fig. 1. A simple architecture of 3-layer fully connected neural network. Circles in each layer represent one neuron

The output is fed into the neuron of next layer. Usually neurons between two adjacent layers are fully connected, and neurons within a single layer are not interconnected. Architecture of a 3-layer neural network is shown in Fig. 1. The problem with this simple neural network is scaling. For example if the input image has spatial size of $32 \times 32$, then one neuron in first hidden layer would have $32 \times 32=1024$ weights. However, if the input size is increased to $200 \times 200$ then one neuron will have $4 \mathrm{~K}$ weights and optimization of these much parameters will lead to over-fitting.

In CNN, each neuron is connected to a small portion of input layer. The neuron computes a value by taking dot product between the weights and small input region. This layer is called CONVolutional layer (CONV layer). The size of the small region is called receptive field. The conv layer have neurons stacked in three dimensions, depth, height and width. The depth of output volume is defined by number of neurons. Height $(H)$ and width $(W)$ is defined by receptive filed or filter size, padding, and stride. The output volume can be calculated using the formula

$$
W=H=\frac{N-F}{\text { stride }}+1 \text {. }
$$

Here $N$ is the input size and $F$ is the filter size. Rectified Linear Unit (ReLU) layer is used to apply non-linearity (activation function) for each element of 3D volume obtained after convolution. An activation function should has maxima and minima (monotonic), must be continuous and differentiable. Pooling layer comes after the ReLU layer, which reduces the size of input volume in spatial domain. The reduction of spatial size is necessary to reduce the number of parameters in subsequent layers as number of neurons increases. This also helps to avoid overfitting and introduce translational invariance in the learned model. At the end of several 
conv+ReLU+pool layers, Fully Connected (FC) layers are used for classifying the input image based on learned features.

\subsection{VGG face topology}

For this study VGG-Face network is used. The architecture of this model is based on the Visual Geometry Group (VGG) deep convolutional neural network proposed in [22]. The main reason behind opting this network is that it is pre trained on a large face dataset of 2.6 million images. It presents comparable accuracy to state of the art networks, which are much deeper than this, on benchmarks like Labelled Faces in Wild (LFW) and YouTube Faces Dataset [22].

VGG-Face net comprises of 37 layers, which are divided in 6 blocks. In each Convolutional Layer (Conv Layer), filter size of $3 \times 3$ is used with stride 1 and padding 1 . First two blocks have 2 convolutional layers each followed by ReLU layers. After 2nd ReLU layer there is max pool layer, which reduces the spatial size of feature map to half. Next three block have three convolutional layers each, followed by ReLU layers and max pool layer after 3rd ReLU layer. Last block comprises of 3 fully connected layers followed by soft-max layer. Input image size for this architecture is $224 \times 224 \times 3$ and it is trained on $2.6 \mathrm{M}$ images with 2622 different classes (identities). Trained network model is available for academic use [23]. The model is implemented in open source MatConvNet library [24].

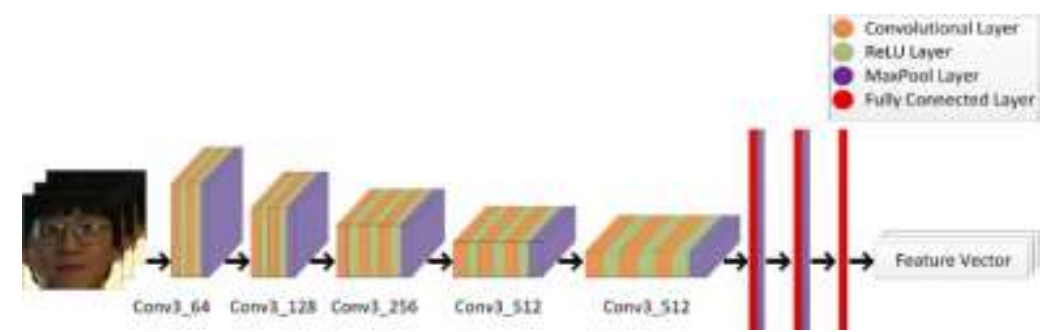

Fig. 2. VGG Face Network architecture. ConvX_ $Y, X$ represents the spatial size of the filter used and $Y$ represents the number of filter used in particular convolutional layer. Feature vector is 4096 dimensional vector

\subsection{Support vector machine}

SVM is a traditional binary classifier used in many different pattern classification applications. Other than pattern classification, it has also been successfully applied to bioinformatics; face detection and recognition; object detection and recognition; handwriting recognition; information and image retrieval; speaker and speech verification [25]. SVM has good generalization properties. This is because it is based on the Structural Risk Minimization (SRM) principal, which is rooted in the statistical learning theory. Supervised learning is used for training the machines with input data labelled with different classes. Principally, SVM finds a hyperplane, given by Formula (2) below, which separates the two classes labelled with $\{-1,1\}$ with maximum margin. Margin is the distance between the two lines, which pass through the data points, which are closest to the opposite class. These data points are called Support Vectors (SV). 


$$
f(x)=\sum_{i=1}^{n} w_{i} x_{i}+b .
$$

The line passing through the SV have equations $w \cdot x_{i}+b \geq 1$ for class +1 and w. $x_{i}+b \leq-1$ for class -1 ,

$$
\begin{gathered}
w \cdot x_{1}+b=1, \\
w \cdot x_{2}+b=-1, \\
w \cdot\left(x_{1}-x_{2}\right)=2, \\
\frac{w}{\|w\|} \cdot\left(x_{1}-x_{2}\right)=\frac{2}{\|w\|} .
\end{gathered}
$$

Maximum margin can be found by minimizing (5), with subject to constraint $y_{i}\left(w \cdot x_{i}+b\right) \geq 1$. This form a Quadratic Programming (QP) problem. World data is usually not separable by the linear classifier. For this reason non-linear kernel functions are used to map data from input space to a higher dimensional space where data can be linear separable.

\subsection{Pre-processing}

All the face images before testing and training are aligned using the dense fiducial points of eyes, eyebrows, nose and mouth, in total 68 points. For fiducial point detection and alignment, Dlib library [26] is used with its python wrapper. Histogram of Oriented Gradients (HOG), image pyramid and sliding window technique is used to find the bounding box for face and [27] is used for fiducial point detection. Images are then align to one common reference face by finding the minimum sum of squared difference between these fiducial points. This process is done by Ordinary Procrustes Analysis, given in

$$
\sum_{i=1}^{68}\left\|y_{i}^{t}-\left(R x_{i}^{t}+T\right)\right\|^{2}
$$

where $R$ is the rotational $2 \times 2$ matrix, $T$ is a translational $2 \times 1$ vector and $y_{i}$ and $x_{i}$ are the coordinates of fiducial points of reference and target images respectively. For cropping the faces, Eye detection is performed using Haar cascaded classifier implemented in Matlab.

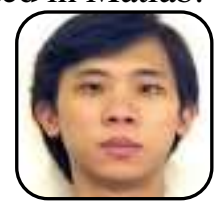

(a)

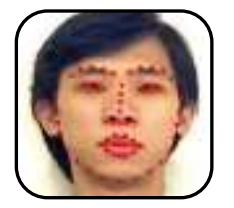

(b)

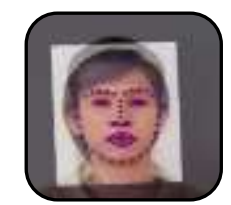

(c)

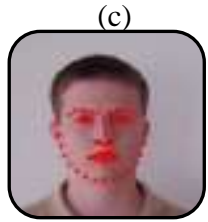

(e)

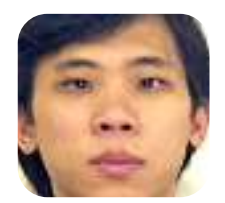

(d)

Fig. 3. Face alignment and crop is represented: the input image (a); shows the 68 landmarks detected (b); represents the layover of reference fiducial points over input image (c); face cropped (d); reference image (e) 
A $224 \times 224$ patch is cropped with respect to line connecting the eye centres such that for every face it should roughly come in the same row. Mean of the data used to train is subtracted from the input images. VGG-Face Net is used to extract the 4096-d feature vector. Support vector machine with linear kernel is used for training, on the feature vectors obtained from convolutional neural network.

\subsection{Visualization}

Fig. 4 shows the 2D t-SNE (t-Distributed Stochastic Neighbour Embedding) [28] plot of the whole database and the features extracted from VGG Face network. The spatial distance indicates the similarity between the images and show strong clustering of images from same class. Although the feature vector, which is 4096-dimensional, is shown in 2D space, it still shows superiority of network in increasing inter-class separability and intra-class compactness.
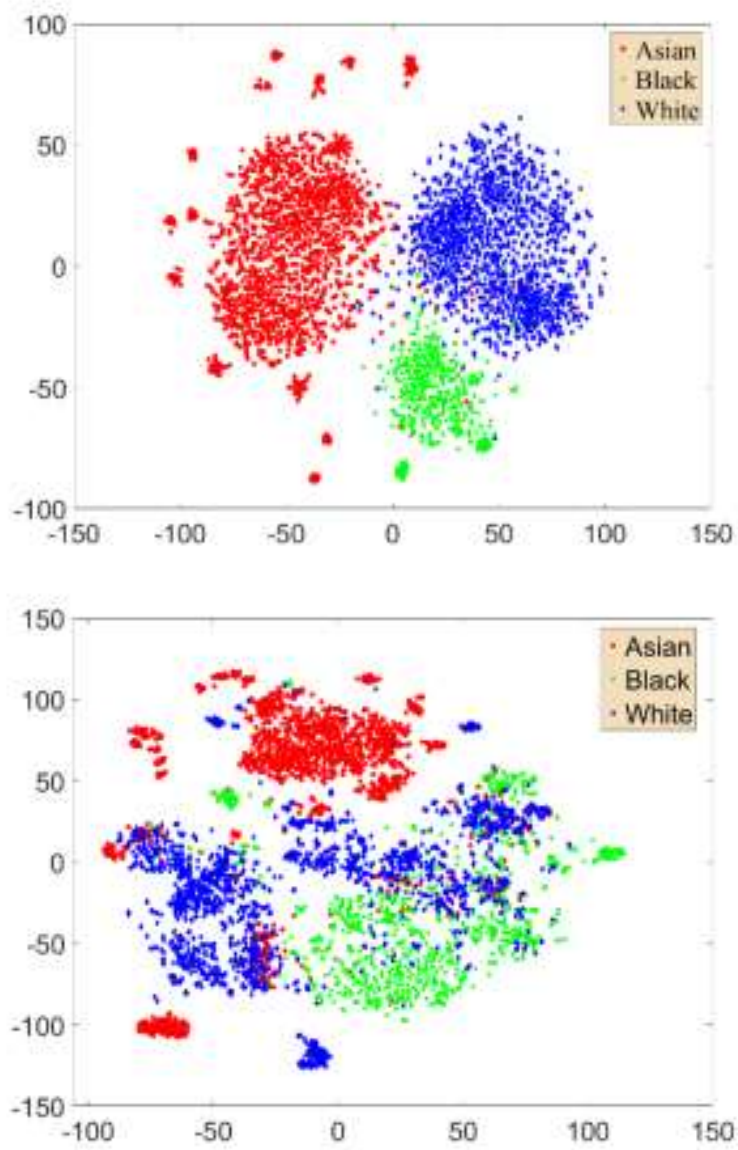

Fig. 4. 2-D t-SNE plot of features obtained from CNN (above) and complete dataset (below) is shown. These plots are generated with $1 \mathrm{~K}$ iterations and perplexity 30 . The face images are resized to $38 \times 38$ 


\section{Experiments and results}

\subsection{Databases}

The proposed process is evaluated on ten different databases including: Computer Vision Lab (CVL) face database [29], Chicago Face Database (CFD) [30], Face Recognition Technology (FERET) database [31], Multi-racial mega resolution (MR2) face database [32], UT Dallas face database [33], Psychological Image Collection at Stirling (PICS) Aberdeen [34], Japanese Female Facial Expression (JAFFE) database [35], CAS-PEAL-R1 [36], Montreal Set of Facial Displays of Emotion Database (MSFDE) [37] and Chinese University of Hong Kong face database (CUFC) [38]. Purpose of using different databases is to evaluate the proposed methodology rigorously and these datasets are publically (without any cost) available. These databases have variations in pose and viewpoints; illumination condition; different expressions; and emotions. Detailed description of the datasets are provided in Table 1.

Table 1. Detailed breakdown of datasets is presented. N/A means that database has No images for that class, N (Normal expression), Var-ill (Variable illumination), S (Smile with

Teeth visible (T) and invisible (NT)), A (Angry), F (Fear), HC (Happy mouth Closed), HO

(Happy mouth Open), Acc. (Accessories), Age. (Aging), Bac. (Background), Dist. (Different viewpoint), Exp. (Expression)

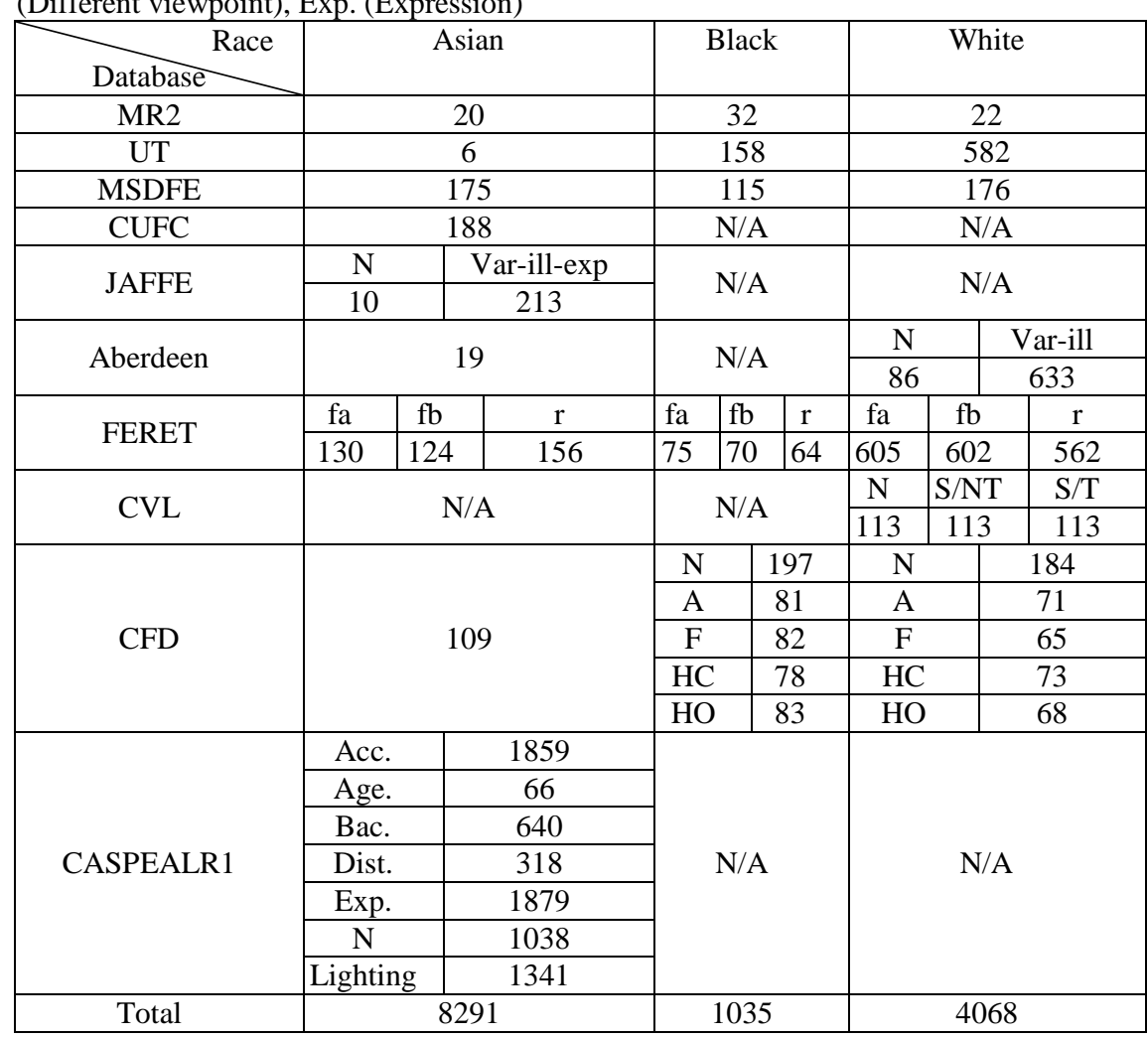




\subsection{Experiments}

The results presented here are obtained using ten-fold cross validation. For each fold, nine databases are used to train linear SVM with five-fold cross validation and remaining one database is used for testing. In all cases, test set and train set are mutually exclusive meaning image of an individual in test is not in the train set, in any case, or vice versa. Although, train or test set may contain more than one image per individual. As most of the databases have different poses, illumination and lighting conditions, neutral face images are only used for training. However, all images are used for testing. This shows the robustness of our technique. Oversampling is used if the number of images for one class is less than others.

Figs 5 and 6 show the results for databases, which have at least two classes. In Aberdeen database there are only two classes Asian and White. Accuracy of Asian class in CFD database is low because there is strong visual resemblance of misclassified persons with Black class. For FERET database separate confusion matrices are shown for three types of sets named "fa", "fb" and " $r$ ". The first set "fa", contains images of subjects with frontal pose, second set "fb" contains images with frontal pose with different expressions and third set, " $r$ " contains images with a pose, head turned by $\pm 15^{\circ}$.
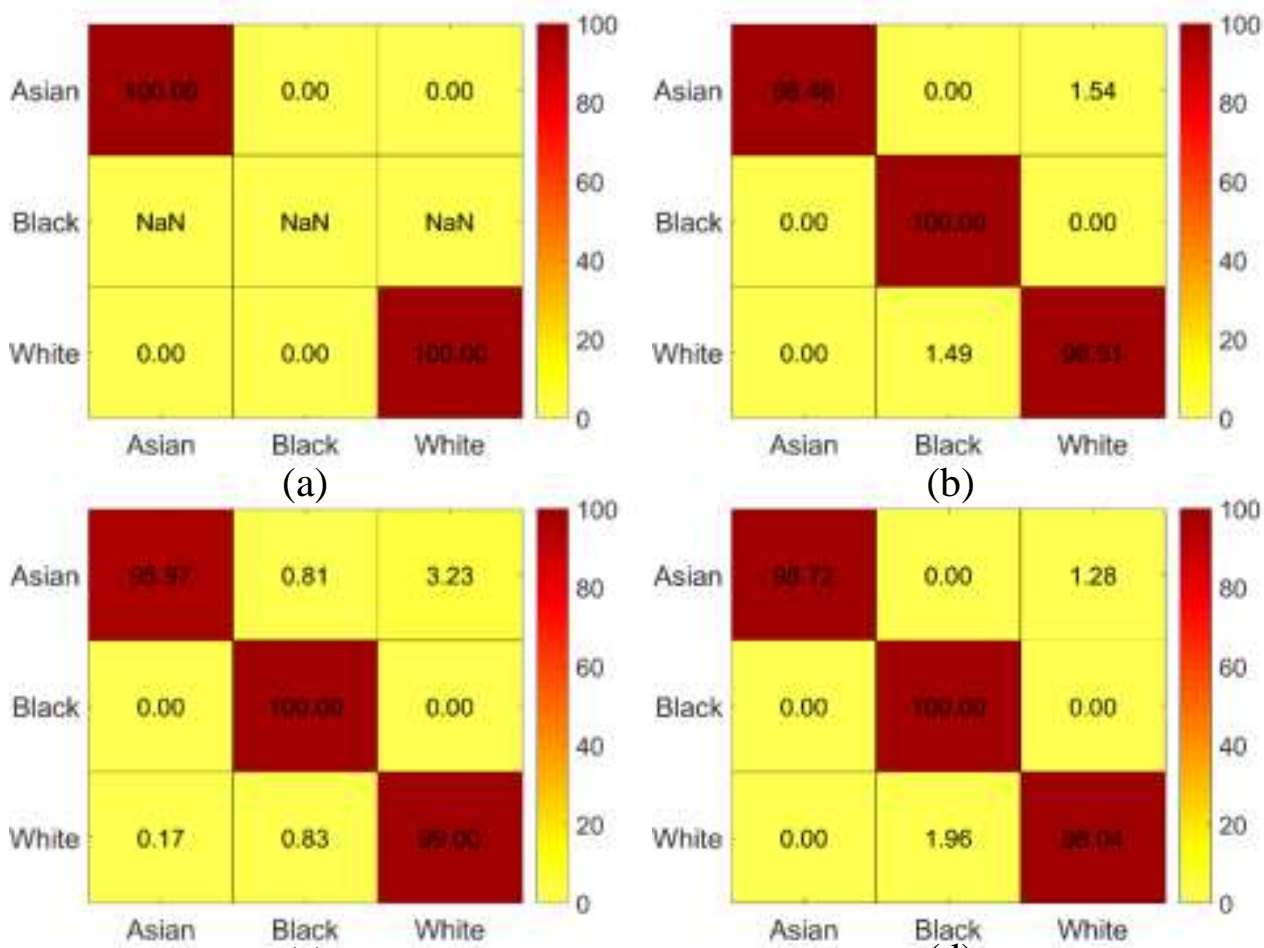

(c)

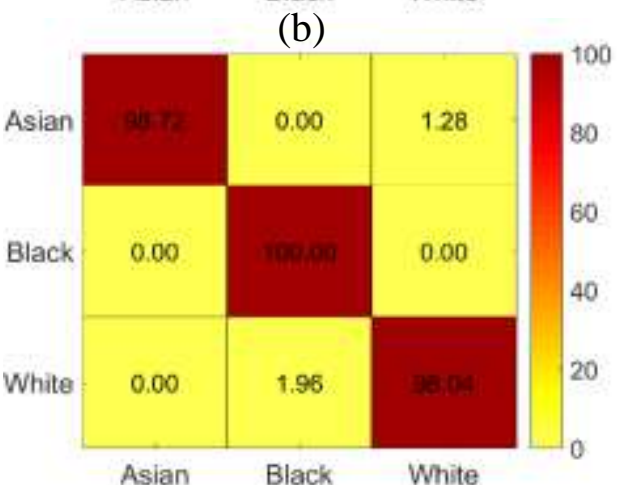

(d)

Fig. 5. Confusion matrices are shown respectively for: Aberdeen (a); FERET fa (b); FERET fb (c); FERET $r(d)$

Classification results for MR2, MSDFE, UT Dallas and CFD database with neutral expressions is shown in Fig. 6. In CFD, for some subjects from Black and White class, there are images with expression. These expression includes angry, fear, 
smile with teeth visible and smile with teeth not visible. Results for these images are shown in Fig. 7.

For CASPEAL database, results are shown in Fig. 8. It includes frontal images with different characteristics; including normal expression; variation in distance between camera and person; wearing different accessories (Sunglasses and Hat); aging (photos of subjects taken with an interval of 6 months); with different illumination conditions; different backgrounds; and different expressions (open mouth, frown, closed eyes, smile and surprise). In case of different illumination conditions, the accuracy is low due to extreme changes in illumination. The illumination conditions are represented in Fig. 3 of [36]. It is observed that the illumination condition in which fluorescent light is below the face of the target; when some parts of the face are not illuminated, the results are erroneous.

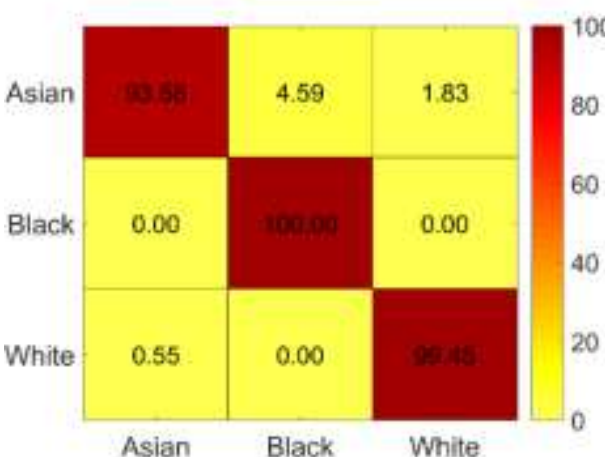

(a)

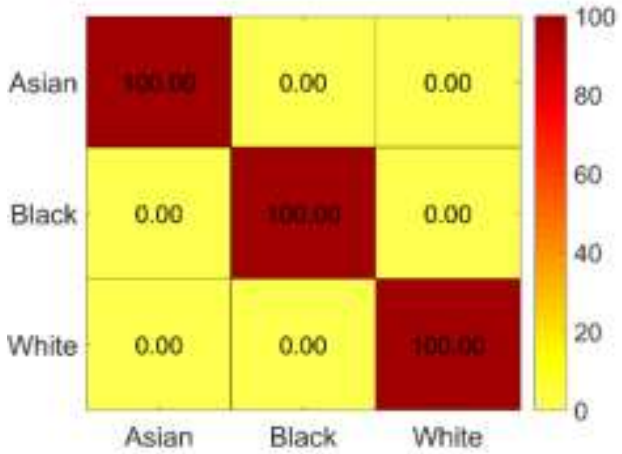

(c)

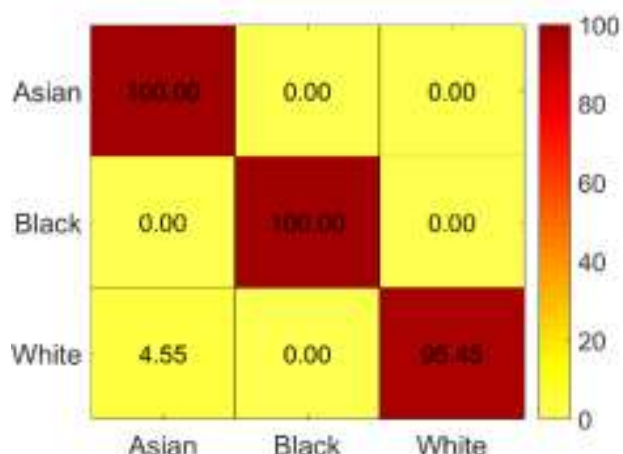

(b)

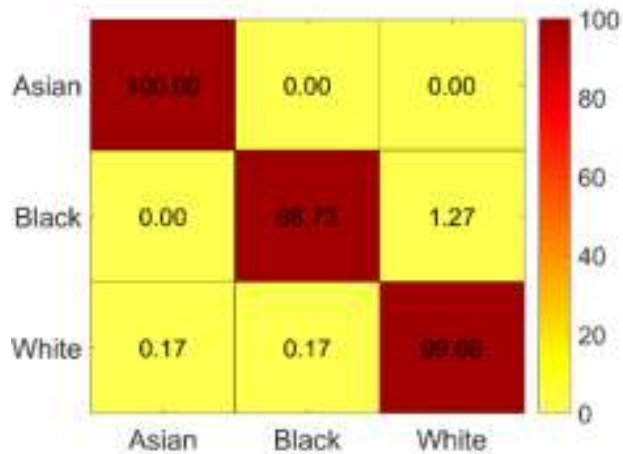

(d)

Fig. 6. Confusion matrices for CFD datasets: with no expression (a); MR2 (b); MSDFE (c); UT Dallas (d) 

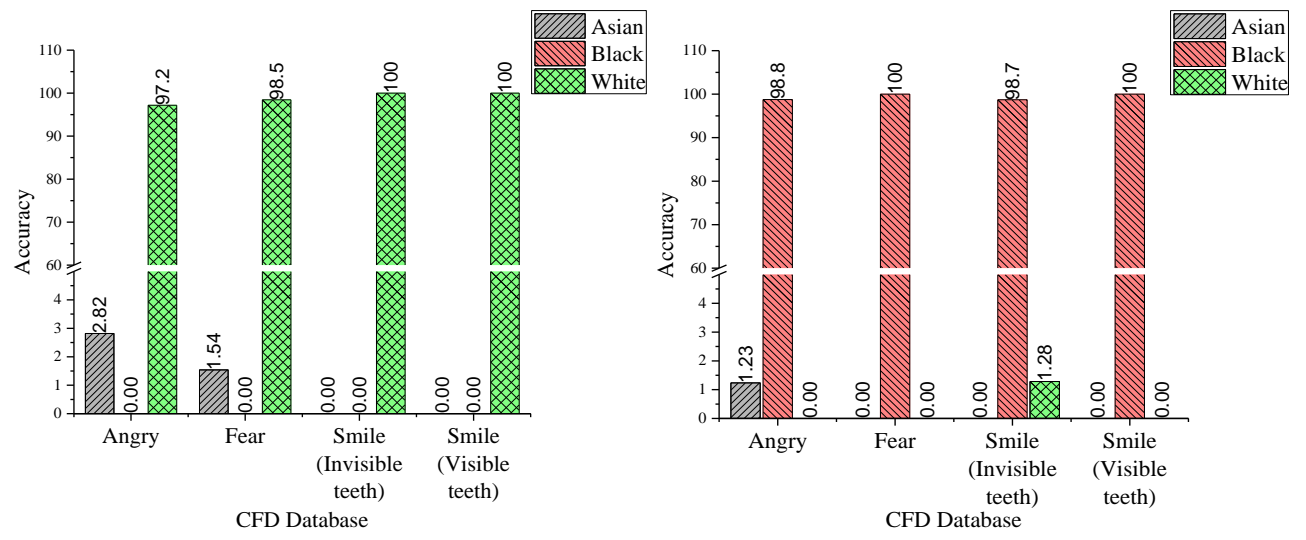

Fig. 7. Results for CFD Database for White and Black subjects, which have different expressions

These results in addition to expression results presented with CASPEAL database strongly suggest that this approach is robust to commonly encountered expressions.

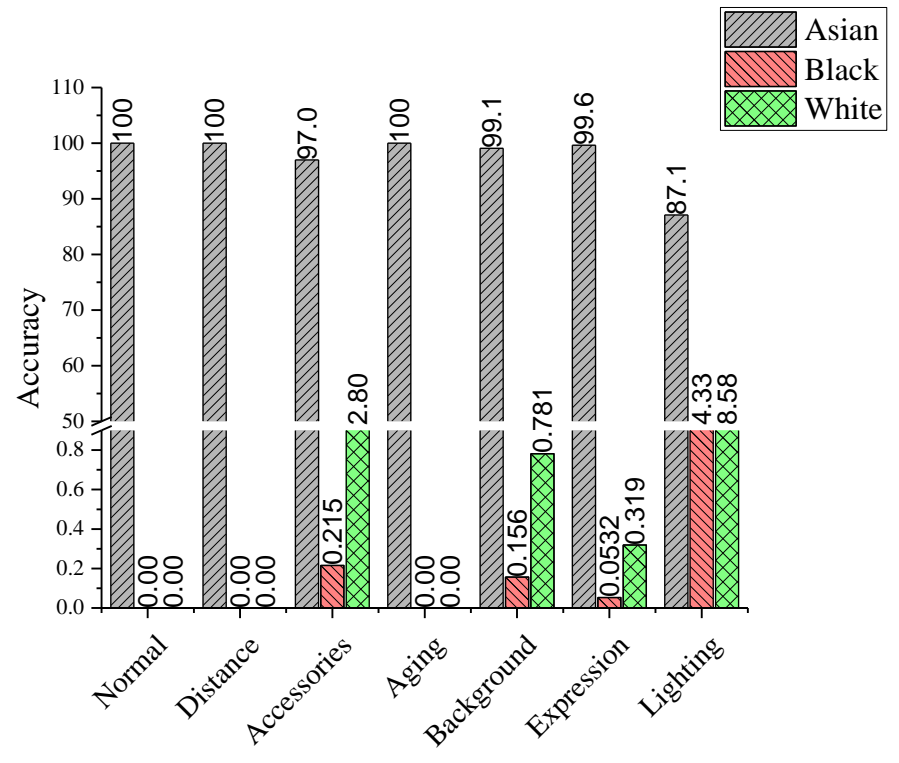

Fig. 8. Results for CASPEAL R1 dataset

\subsection{Comparison}

Table 2 shows comparison between our findings and previous best results in literature. Most of these results are presented on FERET and CASPEAL face database. The results show that our approach performs better than all previous methods. In [2] race classification is taken as two class (Asian/Non-Asian) problem, which is an easier problem than our 3-class classification. They have used images for test and train from same pool of images, which does not include cross database 
problems. In [8], they also used training and testing images from the same database. Although the accuracy in [12] is comparable to our method, the training and test set are not explicitly defined and it is difficult to say whether the images in training and test set are mutually exclusive or not.

Table 2. Comparison with existing methods in literature. *represents the average accuracy our fa, fb and $r$ subsets of FERET database. ** shows the average accuracy our all the datasets

\begin{tabular}{|c|c|c|c|c|}
\hline \multirow{2}{*}{ Method } & \multicolumn{3}{|c|}{ Accuracy } & \multirow{2}{*}{ Database } \\
\cline { 2 - 4 } & Asian (\%) & Black (\%) & White (\%) & \multirow{2}{*}{ FERET } \\
\hline $\begin{array}{c}\text { M a n e s h, G h a h r a m a n i } \\
\text { and T a n [2] }\end{array}$ & 96 & N/A & N/A & FERET \\
\hline G u t t a et al. [8] & 92 & 92 & 92 & FERET \\
\hline M u h a m m a d et al. [12] & 99.47 & 98.99 & 100 & FERET \\
\hline W u, A i and Hu a n g [39] & 95 & 96.1 & 93.5 & FERET+Yale \\
\hline R o o m i et al. [40] & 79.13 & 90 & 90.9 & FERET \\
\hline O u et al. [41] & 83.5 & N/A & N/A & FERET (fa) \\
\hline Ours & $\mathbf{9 8 . 4 6}$ & $\mathbf{1 0 0}$ & $\mathbf{1 0 0}$ & FERET (fb) \\
\hline Ours & $\mathbf{9 5 . 9 7}$ & $\mathbf{1 0 0}$ & $\mathbf{9 9}$ & FERET (r) \\
\hline Ours & $\mathbf{9 8 . 7 2}$ & $\mathbf{1 0 0}$ & $\mathbf{9 8 . 0 4}$ & FERET \\
\hline Ours* & $\mathbf{9 7 . 7 2}$ & $\mathbf{1 0 0}$ & $\mathbf{9 9 . 0 1}$ & All 10 \\
\hline Ours** & $\mathbf{9 8 . 2 8}$ & $\mathbf{9 9 6 6}$ & $\mathbf{9 9 . 0 5}$ & \\
\hline
\end{tabular}

\section{Conclusion}

This paper presents an approach based on Convolutional Neural Network (CNN) to estimate the ethnicity of an individual from facial images. CNN is used here as a standalone feature extractor and linear Support Vector Machine (SVM) is used to classify. Ten different datasets are used for exhaustive experimentation and results presented here outperform the previous techniques. The experiments also indicate that our approach is robust to different illumination conditions, backgrounds, age, gender and various expressions. This work finds its application in Human Computer Interaction (HCI), face recognition, biometric based recognition, surveillance and defence. Future work may include the development of a framework, which can efficiently classify sub-ethnic group, e.g., classifying Koreans from Chinese or Europeans from Americans.

\section{References}

1. F u, S., H. H e, Z. G. H o u. Learning Race from Face: A Survey. - IEEE Transactions on Pattern Analysis and Machine Intelligence, Vol. 36, 2014, No 12, pp. 2483-2509.

2. M a n e sh, F. S., M. Gh a hr a man i, Y. P. T a n. Facial Part Displacement Effect on TemplateBased Gender and Ethnicity Classification. - In: Proc. of 11th International Conference on Control Automation Robotics \& Vision (ICARCV), Singapore, 2010, pp. 1644-1649.

3. $\mathrm{X}$ i a o g u a $\mathrm{g}$, L u., A. K. J a i n. Ethnicity Identification from Face Images. - In: Proc. of SPIE International Symposium on Defense and Security: Biometric Technology for Human Identification, Orlando, Florida, USA, 2004, pp.114-123.

4. T i n, H. H., M. M. S e i n. Race Identification for Face Images. - ACEEE International Journal on Information Technology, Vol. 1, 2011, No 2, pp. 118-120. 
5. D u a n, X. D., C. R. W a n g, X. D. Li u, Z. J. L i, J. W u, H. L. Z h a n g. Ethnic Features Extraction and Recognition of Human Faces. - In: Proc. of 2nd International Conference on Advanced Computer Control (ICACC), Shenyang, Liaoning, China, 2010, pp.125-130.

6. Z h a n g, H., Z. S u n, T. T a n, J. W a n g. Ethnic Classification Based on Iris Images. - In: Proc. of Chinese Conference on Biometric Recognition (CCBR), Beijing, China, 2011, pp. 82-90.

7. X i e, Y., K. L u u, M. S a v vi d e s. A Robust Approach to Facial Ethnicity Classification on Large Scale Face Databases. - In: Proc. of 5th IEEE International Conference on Biometrics: Theory, Applications and Systems (BTAS), Arlington, VA, USA, 2012, pp. 143-149.

8. Gutt a, S., J. R. Hu an g, P. J o n a th o n, H.W e c h s l e r. Mixture of Experts for Classification of Gender, Ethnic Origin, and Pose of Human Faces. - IEEE Transactions on Neural Networks, Vol. 11, 2000, No 4, pp. 948-960.

9. L i n, H., H. L u, L. Z h a n g. A New Automatic Recognition System of Gender, Age and Ethnicity. - In: Proc. of 6th World Congress on Intelligent Control and Automation (WCICA), Dalian, China, Vol. 2, 2006, pp. 9988-9991.

10. Y a n g, Z, H. A i. Demographic Classification with Local Binary Patterns. - In: Proc. of International Conference on Biometrics (ICB), Seoul, South Korea, 2007, pp. 464-473.

11. W a d y, S. H., H. O. A h m e d. Ethnicity Identification Based on Fusion Strategy of Local and Global Features Extraction. - International Journal of Multidisciplinary and Current Research, Vol. 4, 2016, No 2, pp. 200-205.

12. M u h a m m ad, G, M. Hu s s a in, F. Al e n e z y, G. B e bi s, A. M. Mirza, H. A b o al s a m h. Race Classification from Face Images Using Local Descriptors. - International Journal on Artificial Intelligence Tools, Vol. 21, 2012, No 5, pp. 125-149.

13. S a 1 a h, S. H., H. D u, N. A l-J a w a d. Fusing Local Binary Patterns with Wavelet Features for Ethnicity Identification. - In: Proc. of World Academy of Science, Engineering and Technology (WASET), Çanakkale, Turkey, 2013, No 79, pp. 471-477.

14. Krizhevsky, A., I. Sutskever, G. E. Hinton. Imagenet Classification with Deep Convolutional Neural Networks. - In: Proc. of Advances in Neural Information Processing Systems (NIPS), Nevada, USA, 2012, pp. 1097-1105.

15. T a i g m a n, Y., M. Y an g, M. A. R a n z a t o, L. W o lf. Deepface: Closing the Gap to HumanLevel Performance in Face Verification. - In: Proc. of IEEE Conference on Computer Vision and Pattern Recognition (CVPR), Columbus, OH, USA, 2014, pp. 1701-1708.

16. Kim, Y. Convolutional Neural Networks for Sentence Classification. arXiv Preprint arXiv:1408.5882. 25 August 2014.

17. Girsh i c k, R., J. D on a h u e, T. D a r e 11, J. M a lik. Rich Feature Hierarchies for Accurate Object Detection and Semantic Segmentation. - In: Proc. of IEEE Conference on Computer Vision and Pattern Recognition, Columbus, OH, USA, 2014, pp. 580-587.

18. Karpathy, A., G. Toderici, S. Shetty, T. Leung, R. Sukthankar, L. Fei-Fei. Large-Scale Video Classification with Convolutional Neural Networks. - In: Proc. of IEEE Conference on Computer Vision and Pattern Recognition (CVPR), Columbus, OH, USA, 2014, pp. 1725-1732.

19. Ji, S., W. X u, M. Yang, K. Yu. 3D Convolutional Neural Networks for Human Action Recognition. - IEEE Transactions on Pattern Analysis and Machine Intelligence, Vol. 35, 2013, No 1, pp. 221-231.

20. L e v i, G., T. H a s s n e r. Age and Gender Classification Using Convolutional Neural Networks.In: Proc. of IEEE Conference on Computer Vision and Pattern Recognition (CVPR) Workshops, Boston, USA, 2015, pp. 34-42.

21. P i n h e i r o, P. H., R. C o 11 o b e r t. Recurrent Convolutional Neural Networks for Scene Labeling. - In: Proc. of International Conference on Machine Learning (ICML), Beijing, China, 2014, pp. $82-90$.

22. Parkh i, O. M., A. V e d a ld i, A. Z i s s e r m a n. Deep Face Recognition. - In: Proc. of British Machine Vision Conference (BMVC), Swansea, UK, Vol. 1, 2015, No 3, p. 6.

23. http://www.robots.ox.ac.uk/ vgg//software/vgg_face/

24. V e d a ld i, A., K. L e n c. Matconvnet: Convolutional Neural Networks for Matlab. - In: Proc. of 23rd ACM International Conference on Multimedia, Brisbane, Australia, 2015, pp. 689-692.

25. Hearst, M. A., S. T. Dumais, E. Osuna, J. Platt, B. S cholkopf. Support Vector Machines. - IEEE Intelligent Systems and their Applications, Vol. 13, 1998, No 4, pp. 18-28.

26. K in g, D. E. Dlib-ml: A Machine Learning Toolkit. - Journal of Machine Learning Research, Vol. 10, 2009, pp.1755-1758.

27. K a z e m i, V., J. S u 11 i v a n. One Millisecond Face Alignment with an Ensemble of Regression Trees. - In: Proc. of IEEE Conference on Computer Vision and Pattern Recognition (CVPR), Columbus, OH, USA, 2014, pp. 1867-1874.

28. M a a t e n, L. V., G. H i n t o n. Visualizing Data Using t-SNE. - Journal of Machine Learning Research. Vol. 9, 2008, pp. 2579-2605. 
29. Fr a n c, S., P. P e e r, B. B a t a g e $1 \mathrm{j}$, S. J u v a n, J. K o v a c. Color-Based Face Detection in the "15 Seconds of Fame" Art Installation. - In: Proc. of Conference on Computer Vision/ Computer Graphics Collaboration for Model-Based Imaging Rendering, Image Analysis and Graphical Special Effects (MIRAGE), Versailles, France, 2003, pp. 38-47.

30. M a, D. S., J. C o r r e 1 l, B. W i t t e n brin k. The Chicago Face Database: A Free Stimulus Set of Faces and Norming Data. - Behavior Research Methods, Vol. 47, 2015, No 4, pp. 1122-1135.

31. Ph i 11 i p s, P. J., H. M o o n, S. A. R i z vi, P. J. R a u s s. The FERET Evaluation Methodology for Face-Recognition Algorithms. - IEEE Transactions on Pattern Analysis and Machine Intelligence, Vol. 22, 2000, No 10, pp. 1090-1104.

32. Stroh ming er, N., K. Gray, V. Chit u c, J. Heffner, C. S che in, T. B. He a gin s. The MR2: A Multi-Racial, Mega-Resolution Database of Facial Stimuli. - Behavior Research Methods, Vol. 48, 2016, No 3, pp. 1197-1204.

33. M i n e a r, M., D. C. P a r k. A Lifespan Database of Adult Facial Stimuli. - Behavior Research Methods, Instruments, \& Computers, Vol. 36, 2004, No 4, pp. 630-633.

34. H a n c c k, P. Psychological Image Collection at Stirling (Pics). http://pics. psych.stir.ac.uk. 2008 Mar.

35. L y o n s, M. J., J. B u d y n e k, S. A k a m a t s u. Automatic Classification of Single Facial Images. - IEEE Transactions on Pattern Analysis and Machine Intelligence, Vol. 21, 1999, No 12, pp. $1357-1362$.

36. G a o, W., B. C a o, S. S h a n, X. C h e n, D. Z h o u, X. Z h a n g, D. Z h a o. The CAS-PEAL LargeScale Chinese Face Database and Baseline Evaluations. - IEEE Transactions on Systems, Man, and Cybernetics-Part A: Systems and Humans, Vol. 38, 2008, No 1, pp. 149-161.

37. B e a u pré, M. G., N. Ch e ung, U. Hes s. The Montreal Set of Facial Displays of Emotion [Slides]. Available from Ursula Hess, Department of Psychology, University of Quebec at Montreal, PO Box. 2000, 8888.

http://psychophysiolab.com/en/accueil.php

38. W a n g, X., X. T a n g. Face Photo-Sketch Synthesis and Recognition. - IEEE Transactions on Pattern Analysis and Machine Intelligence, Vol. 31, 2009, No 11, pp. 1955-1967.

39. W u, B., H. A i, C. Hu an g. Facial Image Retrieval Based on Demographic Classification. In: Proc. of 17th International Conference on Pattern Recognition (ICPR), Cambridge, England, UK, Vol. 3, 2004, pp. 914-917.

40. Ro omi, S. M., S. L. Virasundarii, S. Selva megala, S. J e e van andha m, D. Harih ar a sudh a n. Race Classification Based on Facial Features. - In: Proc. of 3rd National Conference on Computer Vision, Pattern Recognition, Image Processing and Graphics (NCVPRIPG), Hubli, India, 2011, pp. 54-57.

41. O u, Y., X. W u, H. Q i a n, Y. X u. A Real Time Race Classification System. - In: Proc. of IEEE International Conference on Information Acquisition (ICIA), Hong Kong, China, 2005, pp. 378-383. 